\title{
The STAR-X X-Ray Telescope Assembly (XTA)
}

\author{
Ryan S. McClelland*a, Mark W. Bautz ${ }^{\mathrm{b}}$, Joseph A. Bonafede ${ }^{\mathrm{a}}$, Eric D. Miller ${ }^{\mathrm{b}}$, Timo T. Saha ${ }^{\mathrm{c}}$, \\ Peter M. Solly ${ }^{\mathrm{a}}$ William W. Zhang ${ }^{\mathrm{c}}$ \\ ${ }^{a}$ SGT Inc. 7701 Greenbelt Road, Suite 400, Greenbelt, Maryland 20770, USA \\ ${ }^{\mathrm{b}}$ MIT Kavli Institute, Cambridge, Massachusets, 02109, USA \\ ${ }^{c}$ NASA Goddard Space Flight Center (GSFC), Greenbelt, MD USA 20771, USA
}

\begin{abstract}
The Survey and Time-domain Astrophysical Research eXplorer (STAR-X) science goals are to discover what powers the most violent explosions in the Universe, understand how black holes grow across cosmic time and mass scale, and measure how structure formation heats the majority of baryons in the Universe. To achieve these goals, STAR-X requires a powerful X-ray telescope with a large field of view, large collecting area, and excellent point spread function. The STAR-X instrument, the X-Ray Telescope Assembly (XTA), meets these requirements using a powerful X-ray mirror technology based on precision-polished single crystal silicon and a mature CCD detector technology.

The XTA is composed of three major subsystems: an X-ray Mirror Assembly (MA) of high resolution, lightweight mirror segments fabricated out of single crystal silicon; a Focal Plane Assembly (FPA) made of back-illuminated CCDs capable of detecting X-rays with excellent quantum efficiency; and a composite Telescope Tube that structurally links the MA and FPA. The MA consists of 5,972 silicon mirror segments mounted into five subassemblies called metashells. A meta-shell is constructed from an annular central structural shell covered with interlocking layers of mirror segments. This paper describes the requirements, design, and analysis of the XTA subsystems with particular focus on the MA.
\end{abstract}

Keywords: STAR-X, XTA, X-ray telescope, silicon mirrors, meta-shell, module, NGXO, x-ray optics

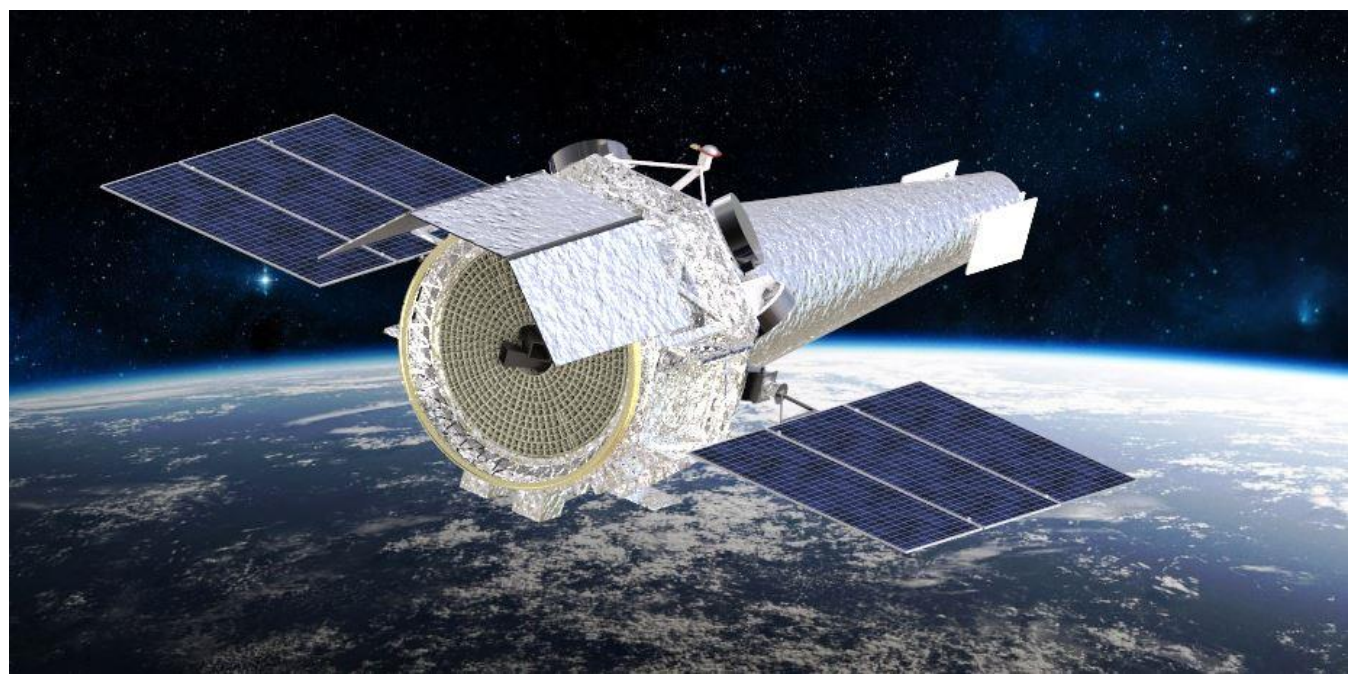

Figure 1. STAR-X will be a powerful X-ray observatory with a wide field of view, large effective area, excellent and uniform PSF, low detector background, and fast slewing spacecraft.

*ryan.s.mcclelland@nasa.gov; phone 1301 286-8615 


\section{INTRODUCTION}

\subsection{STAR-X Science Goals}

The Survey and Time-domain Astrophysical Research eXplorer (STAR-X, Figure 1) will be the most powerful X-ray observatory for discovering transients and surveying wide-areas ever flown [1]. STAR-X fills a critical wavelength gap in the 2020s by providing X-ray coverage when the astronomical landscape will be dominated by wide-area imaging surveys such as the Large Synoptic Survey Telescope (LSST; optical), Wide-Field Infrared Space Telescope (WFIRST; infrared), and the Square Kilometer Array (SKA; radio). STAR-X's power lies in its unique combination of 1) wide field of view, 2) large effective area, 3) excellent and uniform point spread function over the entire field of view, 4) low detector background, and 5) a fast-slewing spacecraft bus. Table 1 shows the key mission and instrument requirements. It addresses the broad and diverse science questions more typical of a Flagship-class mission. STAR-X's three primary science goals are to:

- Discover what powers the most violent explosions in the Universe. STAR-X's observations can uniquely characterize the counterparts of gravitational wave sources from compact binary mergers, identify the progenitors of supernovae, and discover the very faint off-axis afterglows from Gamma-Ray Bursts (GRBs).

- Understand how black holes grow across cosmic time and mass scale. STAR-X will uncover previously hidden black holes (BHs) across the entire mass range, from 1-1010 M $\odot$, and over 12.5 billion years of cosmic time. STAR-X will constrain models of galaxy and black hole formation, and will help pin down the formation channel of gravitational wave sources.

- Measure how structure formation heats the majority of baryons in the Universe. STAR-X will directly witness the growth of cosmic structure, both within individual nearby galaxy clusters and through their assembly across 11 billion years of cosmic time. As a result, STAR-X will calibrate the X-ray emission from these sources for precision cosmology.

Table 1. STAR-X requirements lead to a powerful observatory which addresses broad and diverse science questions.

\begin{tabular}{|c|c|c|c|c|c|c|}
\hline \multicolumn{7}{|c|}{ Instrument and Mission Requirements } \\
\hline \multirow{8}{*}{ 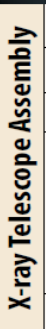 } & Energy Band & \\
\hline & Field of View & \multicolumn{5}{|l|}{\begin{tabular}{|l|l}
$0.5-6 \mathrm{keV}$ \\
$1.0 \mathrm{deg}^{2}$
\end{tabular}} \\
\hline & Point Spread Function & \multicolumn{5}{|c|}{5 arcsec half-power diameter over entire FOV } \\
\hline & \multirow{4}{*}{ Effective Areas $\left(\mathrm{cm}^{2}\right)$} & Energy (keV) & 0.5 & 1 & 4 & 6 \\
\hline & & 0n-axis & 1270 & 1800 & 250 & 45 \\
\hline & & $0.25^{\circ}$ off-axis & 962 & 1360 & 140 & 17 \\
\hline & & $0.50^{\circ}$ off-axis & 706 & 992 & 70 & 7 \\
\hline & \multicolumn{2}{|c|}{ Spectral Resolution (FWHM) (eV) } & 70 & 80 & 120 & 150 \\
\hline \multirow{7}{*}{$\frac{\overline{0}}{\frac{\tilde{n}}{\Sigma}}$} & Orbit & \multicolumn{5}{|c|}{$5^{\circ}$ inclination, $600 \mathrm{~km}$, circular } \\
\hline & Transient Event Alert & \multicolumn{5}{|c|}{$\begin{array}{l}\text { Within } 30 \text { sec of on-board detection, through TDRSS } \\
\text { ( } 90 \% \text { of the time) }\end{array}$} \\
\hline & Response to To0 & \multicolumn{5}{|c|}{ Within 60 mins of receipt ( $90 \%$ of the time) } \\
\hline & Slew and Repoint & \multicolumn{5}{|c|}{$120^{\circ}$ in less than 5 mins; $0.5^{\circ}$ in $24 \mathrm{sec}$} \\
\hline & Astrometric Knowledge & \multicolumn{5}{|c|}{$2 \operatorname{arcsec}(3 \sigma)$} \\
\hline & Observing Efficiency & \multicolumn{5}{|l|}{$>66 \%$} \\
\hline & Launch Readiness Date & \multicolumn{5}{|l|}{ May 31,2023} \\
\hline
\end{tabular}

\subsection{STAR-X Instrument: The X-Ray Telescope Assembly}

STAR-X's science program is enabled by the X-ray Telescope Assembly (XTA) instrument. The XTA's combination of large field of view (FOV), large collecting area, and excellent point spread function (PSF), will make it a powerful survey X-ray telescope for discovering faint sources over large (hundreds of square degree) areas of the sky and for detecting diffuse emission. The XTA is composed of three major components (Figure 2): an X-ray Mirror Assembly (MA) of high resolution, lightweight mirror segments fabricated out of single crystal silicon; a Focal Plane Assembly (FPA) made of back-illuminated CCDs capable of detecting X-rays with excellent quantum efficiency (QE) over 
bandpass; and a composite Telescope Tube that structurally links the MA and FPA. Each of these components is detailed below. The XTA block diagram and key parameters are shown in Figure 3.

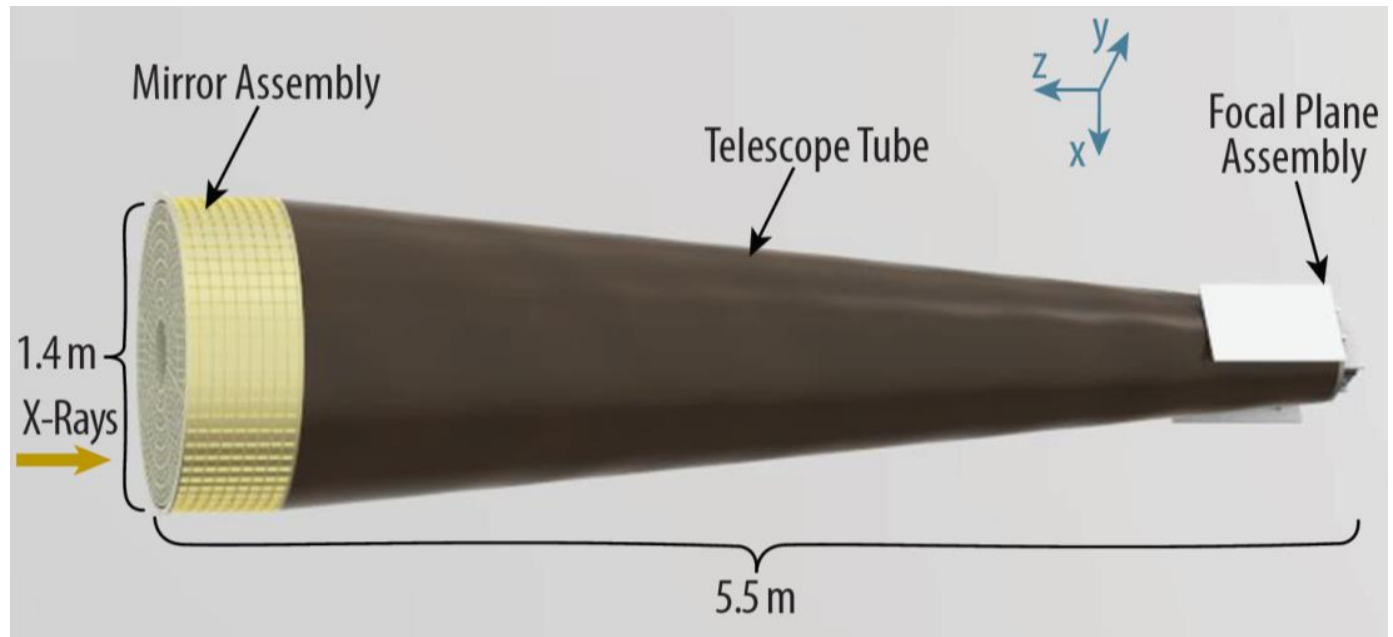

Figure 2. STAR-X will be a powerful X-ray observatory with a wide field of view, large effective area, excellent and uniform PSF, low detector background, and fast slewing spacecraft.

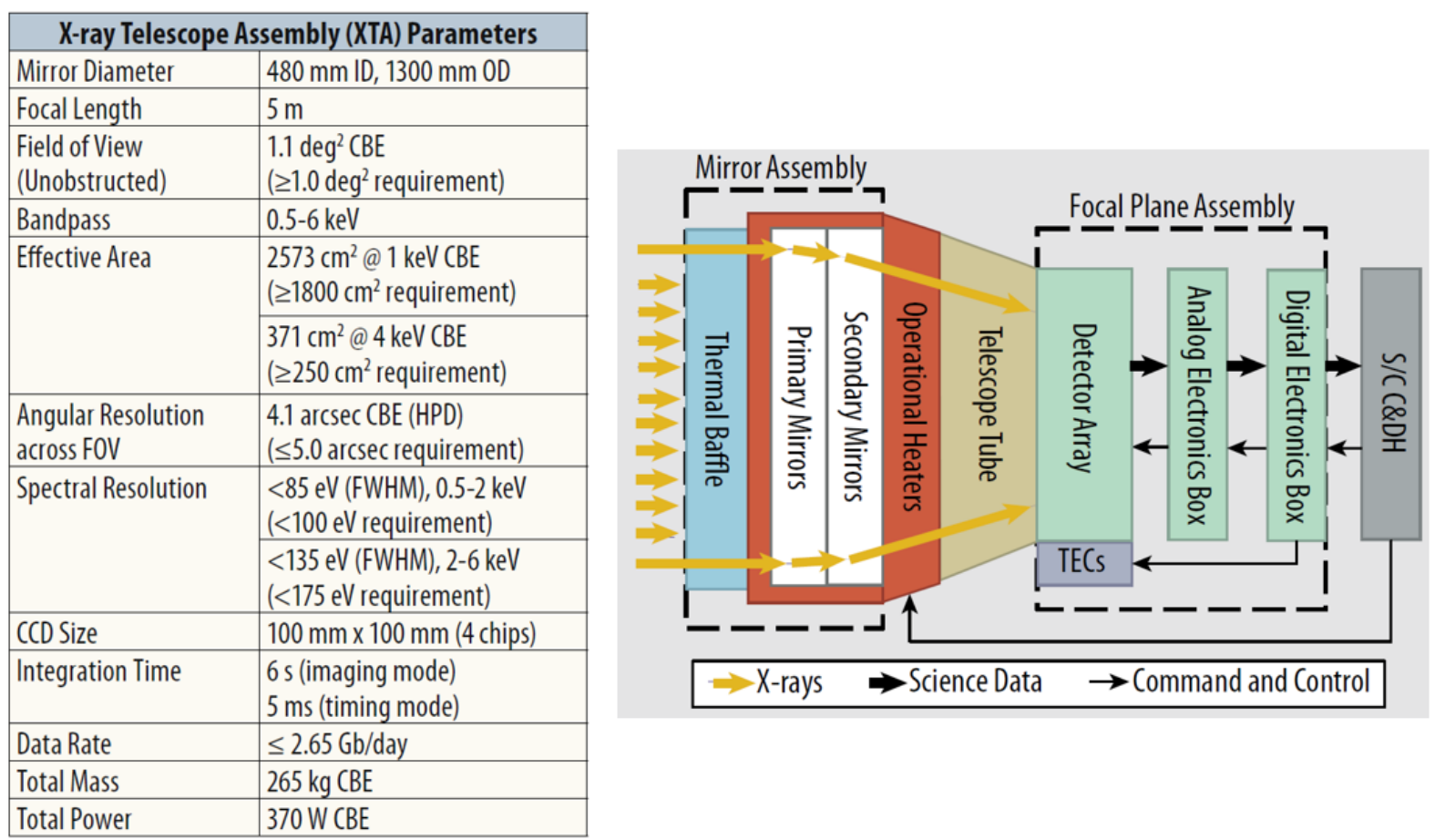

Figure 3. Key parameters of the XTA (left). Block diagram showing clean functional partitioning and simple interfaces (right).

\subsection{STAR-X Mission}

The STAR-X mission design is simple, consisting of a spacecraft that provides structural support, power, telemetry, and commanding to the mission's single instrument, the XTA. The spacecraft bus (Figure 4) maximizes use of off-the-shelf, legacy, and built to-print components. The ground system leverages existing infrastructure and proven data handling systems in GSFC's Multi-Mission Mission Operations Center (MMOC). STAR-X launches on a Falcon 9 v1.1 into a $600 \mathrm{~km}$ low-earth circular orbit at $5^{\circ}$ inclination to reduce background radiation and maximize observing efficiency. 


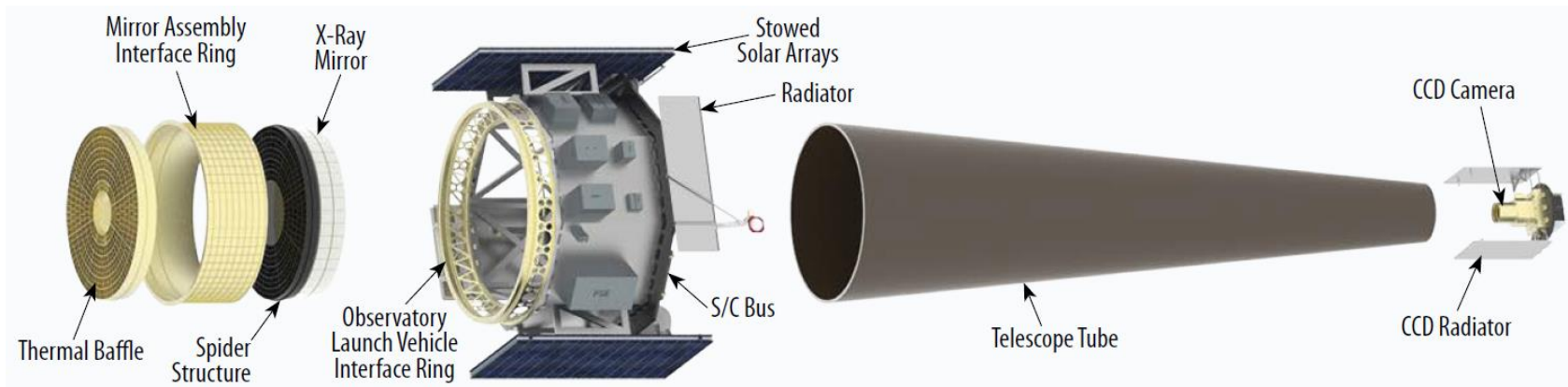

Figure 4. The STAR-X spacecraft bus provides structural support, power, telemetry, and commanding to the XTA.

\section{MIRROR ASSEMMBLY}

The STAR-X Mirror Assembly (MA) consists of thousands of co-aligned polished silicon mirror segments grouped into five optical sub-assemblies called meta-shells, which focus the x-ray photons, and the associated structures, thermal control components, stray light baffles. The X-ray optics technology is being matured by the Next Generation X-Ray Optics (NGXO) group at NASA GSFC with funding from the NASA ROSES Strategic Astrophysics Technology (SAT) program [2].

\subsection{Optical Design}

STAR-X uses a modified Walter-Schwarzschild optical design [3]. The sagittal depth of each mirror is modified slightly to minimize off-axis image distortion at the expense of a slight defocusing of on axis images. Further improvement of off-axis images is achieved by limiting the length of the primary and secondary mirrors in the optical axis direction to $100 \mathrm{~mm}$ and by placing each parabolic-hyperbolic intersection on an optimal principal surface. The optical design requires 101 concentric mirror shells grouped into five sub-assemblies, called meta-shells as shown in Table 2. The optical design is implemented using a segmented approach where each primary or secondary mirror shell is split into many small segments in the azimuthal direction, as shown in Figure 5, leading to 5,972 mirror segments in the design.

Table 2. The STAR-X MA consists of 101 shells/layers of mirror segments grouped into five meta-shells. The outer meta-shells contribute more low-energy effective area, while the inner contribute more high-energy effective area.

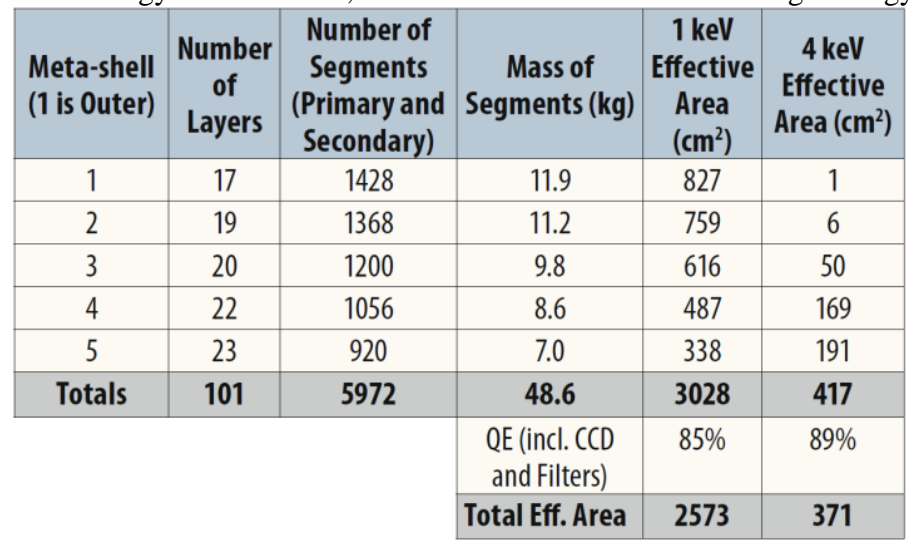

\subsection{Mirror Segments}

The mirror substrate is a piece of single crystal silicon fabricated with a process similar to the industrial silicon wafer production process [4]. The fabrication process starts with a block of single crystal silicon that is ground into a conical surface approximating the parabolic or hyperbolic optical design surface. The conical-shaped surface is sliced off the silicon block. Then, the sliced segment is etched in a hydrofluoric-nitric-acetic solution to remove any crystal structure damage caused by the grinding and slicing processes, resulting in a substrate that is a single crystal. This conical-shaped segment is stressed into a cylindrical shape and polished on a cylindrical polisher for approximately 40 hours to achieve 
the required optical figure and micro-roughness. The segment is then coated with $10 \mathrm{~nm}$ of iridium to enhance its X-ray reflectivity.

\subsection{Meta-shells}

STAR-X's 101 concentric shells are grouped within the MA into five meta-shells (Table 2). A meta-shell is constructed from an annular central structural shell covered with interlocking layers of mirror segments (Figure 5). The structural shell is made of silicon, matching the mechanical and thermal properties of the mirror segments. Each mirror segment is aligned and bonded with four silicon spacers machined in-situ under the guidance of optical metrology [2]. As three points describe the location and orientation of a plane, so four points describe the location and orientation of a cylinder or cone. A mirror segment mounted on four points has its four most crucial degrees of freedom (dof) constrained, namely de-center ( 2 dof), pitch, and yaw. The remaining two degrees of freedom are roll, which has no effect on the focus quality due to rotational symmetry, and de-space, which is orders of magnitude less sensitive. Roll and de-space are constrained only by friction at the four posts and are set by alignment stops before bonding. Once the four spacers are iteratively machined and optically verified, a small amount of adhesive is applied to each spacer and the mirror is placed on them. Gentle vibration settles the mirror into alignment. Once the adhesive cures, the mirror segment is permanently bonded. Several interlocking layers of mirror segments complete the meta-shell. The resulting meta-shell has the stiffness and rotational symmetry similar to a traditional full shell optic, but with an order of magnitude greater collecting area due to the many layers of mirror segments.
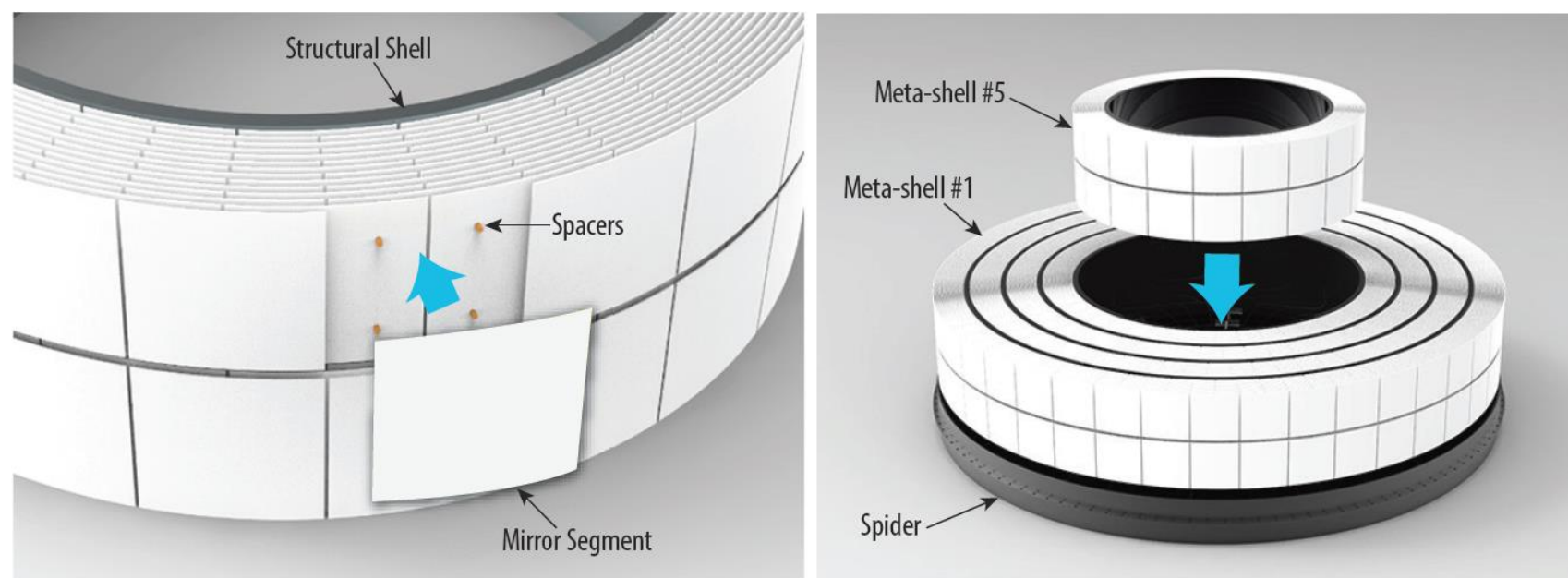

Figure 5. Each mirror segment is aligned and integrated with four silicon spacers to a structural shell, forming a meta-shell of hundreds of mirror segments (left). Multiple meta-shells are co-aligned and mounted together on a spider structure to complete the optical assembly (right).

\subsection{Stray Light Mitigation}

As part of the meta-shell construction process, two annular-shaped pieces of silicon are bonded to the back (convex side) of each mirror segment as internal baffles [3]. Combined with 62-mm long cylindrical baffles integrated to the spider structure, these baffles prevent X-rays outside of the $1 \mathrm{deg}^{2}$ FOV from reaching the focal plane and generating ghost images.

\section{FOCAL PLANE ASSEMBLY}

The Focal Plane Assembly (FPA) consists of a CCD detector array, an Analog Electronics Box (AEB), a Digital Electronics Box (DEB), and associated structures and radiators as shown in Figure 6. The AEB provides CCD readout control, analog signal processing, and digitization. The DEB controls the AEB, receives and extracts X-ray event data from the digitized CCD pixel data stream, and interfaces with the spacecraft command and data handling $(\mathrm{C} \& \mathrm{DH})$ system. These subsystems, including their architecture and major components, have heritage from ASCA, Chandra, and Suzaku. 

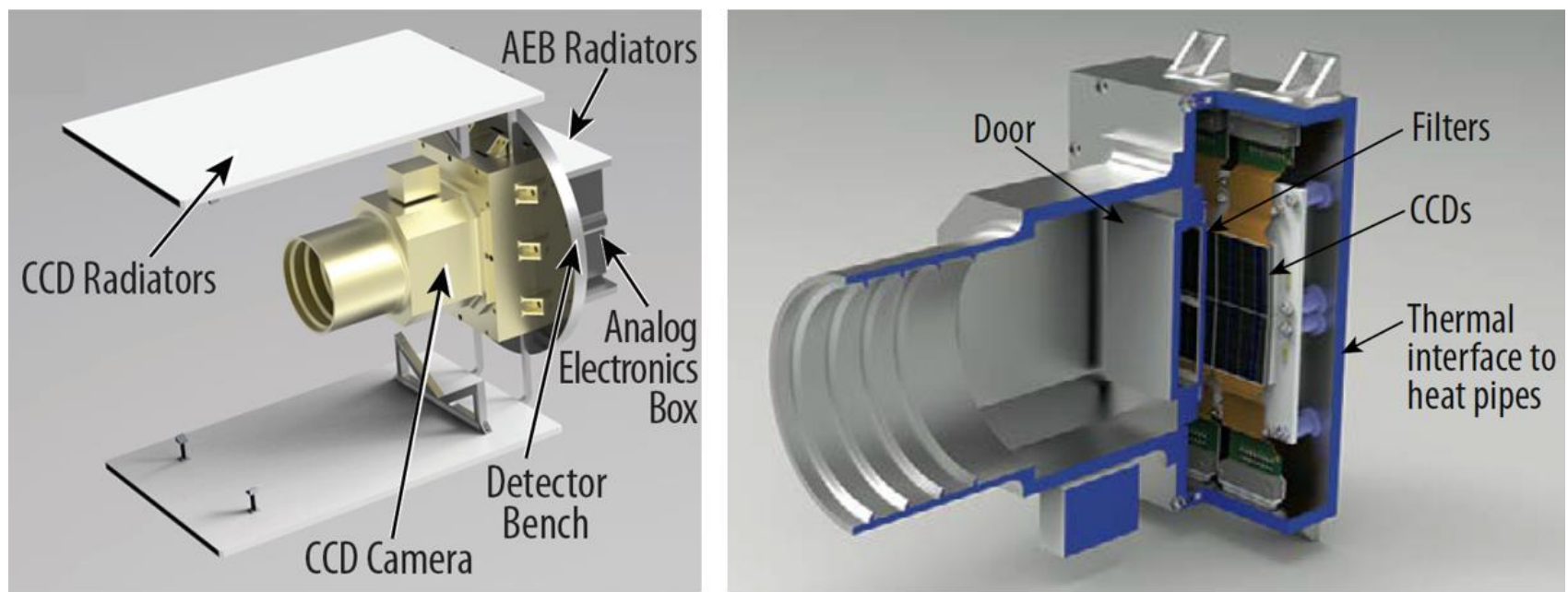

Figure 6. The Focal Plane assembly includes the CCD camera (aka Detector Housing) and associated electrical, thermal, and structural components (left). The CCD chips and thermoelectric coolers are located inside the Detector Housing volume which also includes filters, a vent, and a high reliability door (right).

\subsection{CCD Detector Array}

The CCD chips for the Detector Array (DA) are made by the Massachusetts Institute of Technology (MIT) Lincoln Laboratory. The DA is comprised of four 3200x3200 pixel CCDs arranged in a 2x2 array $100 \mathrm{~mm}$ on a side. The STAR$\mathrm{X}$ CCID-86, a simplified version of a device produced for USAF/Hawaii Pan-STARRS, is a 50x50 mm four-sided abuttable device comprising 16 on-chip 'cells' with a 90\% fill factor. Each cell contains 800x800 15x15 $\mu \mathrm{m}$ pixels, and a dedicated output amplifier. For STAR-X, these cells are read out using $3 \times 3$-pixel $(45 \times 45 \mu \mathrm{m})$ noiseless, on-chip binning to sample the $5 \operatorname{arcsec}(120 \mu \mathrm{m})$ PSF. Four cells of each detector are read out in parallel in $360 \mathrm{~ms}$, with a standard integration time of $6 \mathrm{~s}$ (this affords only $6 \%$ out-of-time events, a scheme proven on XMM-Newton/EPIC-pn). A timing mode with an integration time of $5 \mathrm{~ms}$ is also available. An Optical Blocking Filter, consisting of $50 \mathrm{~nm}$ of aluminum ( $\mathrm{Al}$ ), is directly deposited on the CCD chips; this is supplemented by an additional $20 \mathrm{~nm}$ of $\mathrm{Al}$ on a Contamination Blocking Filter (CBF).

\subsection{Detector Housing}

In addition to the focal plane, the Detector Housing contains an array of thermoelectric coolers (TECs) and a CBF. These elements are launched under vacuum, and the housing features a door assembly and vent system with a commandable valve. The vent system allows the Detector Housing to be evacuated or re-pressurized during ground test with the instrument integrated in the spacecraft, and also provides a path to space for any contaminants. Detector Housing contamination control functions are detailed in Section 6.

\subsection{Operational Modes}

STAR-X has two instrument modes: imaging and timing. In imaging mode, the most common STAR-X prime mission science mode, the CCD array is read out every $6 \mathrm{sec}$ by the AEB and the data is processed by the DEB. At the end of each $6 \mathrm{sec}$ interval, the DEB delivers a list of photons to the spacecraft $\mathrm{C} \& \mathrm{DH}$ computer.

In timing mode, a portion of each CCD detector, on which the source of interest is imaged, is read with $5 \mathrm{~ms}$ time resolution (but limited spatial resolution). This mode is used to observe very bright sources or rapidly varying sources.

\section{XTA STRUCTURE SUB-SYSTEM}

The primary XTA structural components are an interface ring (Figure 7), telescope tube (Figure 2), and detector bench (Figure 6). The aluminum interface ring provides three bolt circles for mounting the spider, unheated thermal baffle, and telescope tube. A fourth bolt circle on the exterior of the interface ring provides the mechanical interface to the spacecraft. The telescope tube provides the mechanical connection between the MA and FPA. Similar to the Chandra Optical Bench [5], it is fabricated from composite facesheets with an Al honeycomb core for lightweight, high stiffness, and low coefficient of thermal expansion (CTE). The telescope tube provides mounting for the FPA, including the CCD 
radiators and detector bench. The detector bench, also made from composite facesheets with Al honeycomb core, provides mounting for the DH and AEB. The XTA structure has been sized and verified by finite element analysis to meet structural requirements.

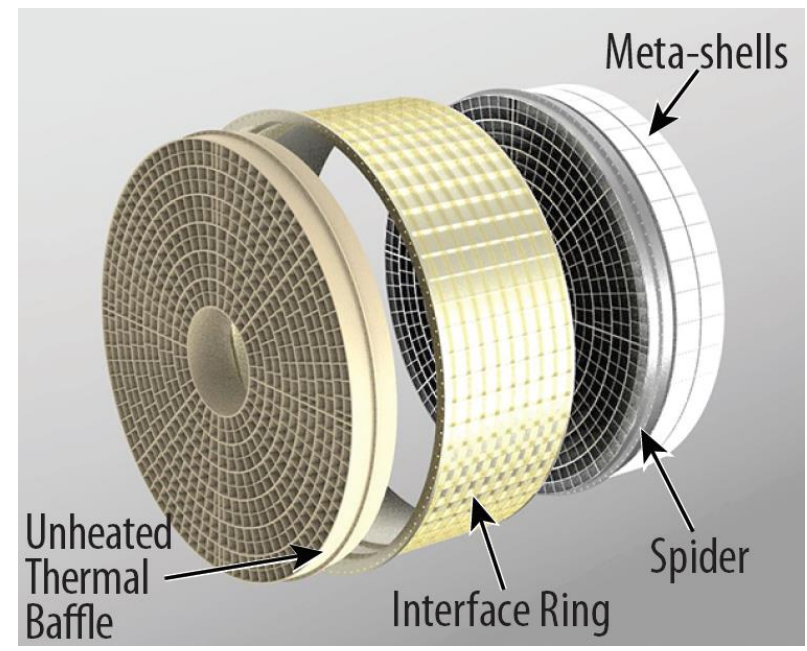

Figure 7. The Mirror Assembly includes the Spider structure with meta-shells, and unheated thermal baffle, and an interface ring which provides mounting for the telescope tube and spacecraft bus.

\section{XTA THERMAL CONTROL SYSTEM}

The instrument thermal control system maintains MA and CCD operating temperatures. The MA is maintained at $20^{\circ} \pm 1.5^{\circ} \mathrm{C}$ to meet thermal distortion requirements (Figure 8). Due to silicon's low CTE $(2.5 \mathrm{ppm} / \mathrm{K}$ ) and high thermal conductivity $(150 \mathrm{~W} / \mathrm{mK})$, the meta-shells are relatively insensitive to thermal disturbances. Heat radiated to space is minimized by the low-emissivity iridium coating on the mirror segments (also required for X-ray reflectivity) and the thermal baffles (heated and unheated), which limit the view factor of the mirror segments to space. Heat lost to space is replaced by operational heaters on the heated thermal baffle vanes, interface ring, and a $1.5 \mathrm{~m}$ section of the telescope tube nearest the MA.
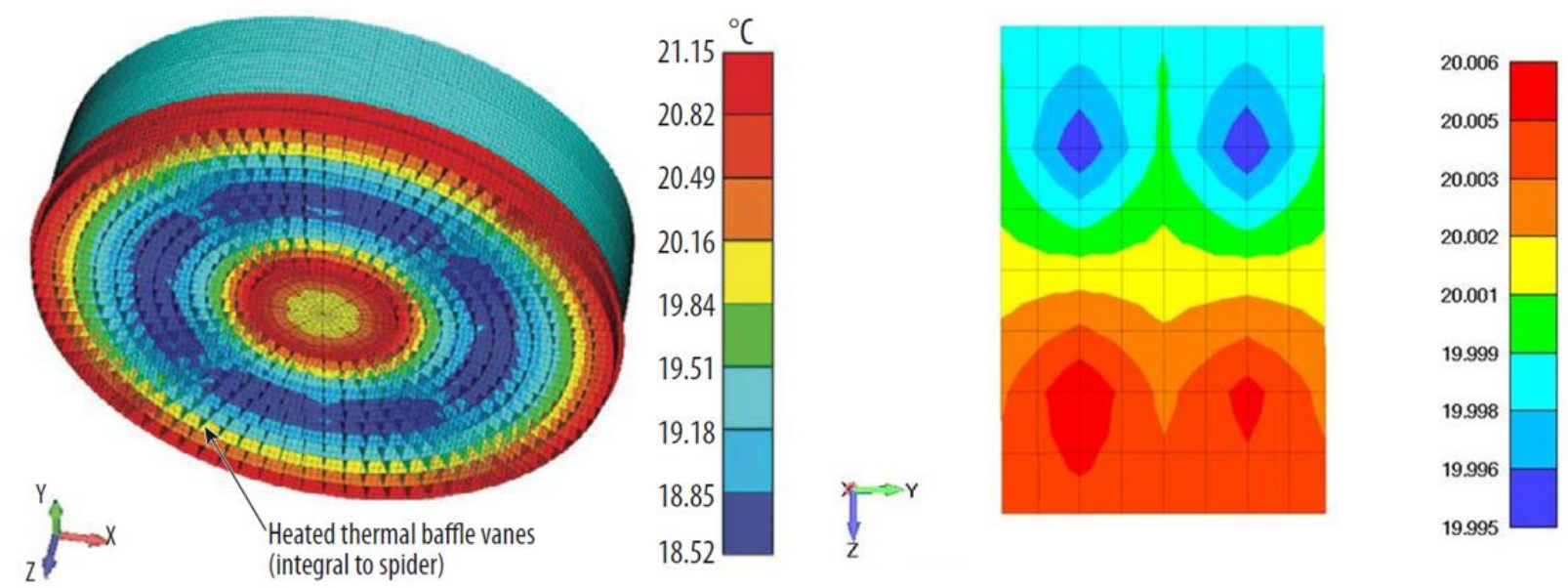

Figure 8. Detailed Structural Thermal Optical Performance (STOP) analysis was used to verify the MA thermal control system meets requirements (left). The gradients over individual mirror segments, which drive focusing performance degradation, are minimized by the high thermal conductivity and low CTE of single crystal silicon.

The CCD focal plane is actively cooled by eight two-stage, space-qualified TECs supplied by Marlow Industries (Appendix J.12). TEC current is controlled to provide a stable focal plane temperature of $-70^{\circ} \pm 0.5^{\circ} \mathrm{C}$, which reduces dark current and susceptibility to radiation damage so performance does not degrade throughout the mission lifetime. 
TEC hot sides are in thermal contact with the body of the Detector Housing, which is maintained at a temperature of $40^{\circ} \mathrm{C}$. Heat is carried away from the Detector Housing via constant conduction heat pipes (CCHPs) to two radiator panels facing $90^{\circ}$ away from the sun, which maximizes the view factor to deep space from low earth orbit. The AEB, is cooled by two small attached radiator panels. The telescope tube exterior is covered with 25-layer MLI to insulate it from the space environment and isolate it from the surrounding S/C bus. Analysis shows telescope tube temperature changes less than $3^{\circ} \mathrm{C}$ throughout the STAR-X orbit, meeting stability requirements.

\section{XTA CONTAMINATION CONTROL}

The main contamination concern for STAR-X is molecular contaminants condensing on the CCDs and absorbing X-ray photons, thereby reducing the effective area, an issue that significantly affects the Chandra mission [6]. STAR-X follows contamination mitigation approaches used successfully on XMM-Newton/EPIC and Swift/XRT, which have not been affected by contamination.

The Detector Housing has a vent that provides a path to space for any contaminants that may outgas within the detector volume, and a Contamination-Blocking Filter $(\mathrm{CBF})$ that isolates the cold detectors from any contaminants that may outgas from the interior of the XTA. The CBF is maintained between 0 and $20^{\circ} \mathrm{C}$ to ensure contaminants do not condense on the filter. The $\mathrm{CBF}$ is coated with $20 \mathrm{~nm}$ of $\mathrm{Al}$ to provide additional UV/optical blocking for the detectors, protect the filter from atomic oxygen, and minimize radiative-coupling between the CBF and cold focal plane. Contaminants may also be evaporated from the detectors on-orbit by reversing the TEC current to warm the CCDs to $+30^{\circ} \mathrm{C}$.

The telescope tube is lined with an aluminum film that prevents contaminants outgassed by the composite material from reaching the interior of the XTA; this approach was used by XMM-Newton to effectively stop contamination. Any contaminants within the XTA are vented via a labyrinth vent to prevent collection on the detectors.

\section{XTA INTEGRATION AND TESTING}

The XTA is integrated, tested, and qualified independently of the spacecraft bus before being delivered to observatory integration and testing (I\&T). A protoflight approach is used for all of the components except for the detector electronics, which employ Engineering Test Units (ETUs). Each major component is performance tested, environmentally tested in accordance with Goddard Environmental Verification Standards (GEVS), then performance tested again before being integrated into the next level of assembly. First, the mirror segments are individually qualified by interferometrically measuring optical figure and micro-roughness. During meta-shell integration, the focus quality, alignment, and bond strength are verified as each mirror segment is installed. The completed meta-shell is then X-ray tested under vacuum at GSFC's $600 \mathrm{~m}$ beam line. On- and off-axis PSF and effective areas at several energies are measured. Next the meta-shell is subject to environmental tests including vibration and shock. MA integration brings together the five meta-shells as well as structural and thermal components. The meta-shells are co-aligned using a Centroid Detector Assembly (CDA) with a retro-reflecting flat mirror, identical to the approach used by Chandra [7]. Following integration, the MA undergoes X-ray performance, alignment, and environmental testing (thermal vacuum, vibration, shock, acoustic) before being delivered to XTA integration. The detector system (Detector Housing, AEB, DEB) is tested, calibrated, and qualified at MIT before being delivered to XTA integration at GSFC. Environmental tests of the detector system include thermal vacuum, vibration, and EMI/EMC. XTA integration brings together the MA, telescope tube, and FPA (which includes the detector system). The XTA undergoes alignment and comprehensive performance testing, followed by environmental testing, including thermal vacuum, vibration, shock, and acoustic. After final qualification, the XTA is delivered to observatory I\&T. 


\section{POINT SPREAD FUNCTION ERROR BUDGET}

The optical design meets the angular resolution requirement by trading on-axis PSF for a minimum PSF over the FOV, resulting in a uniform design PSF of 3 arcsec [3]. Other sources of error include imperfections of the mirror polishing, coating, and mounting, as well as system-level effects from thermal distortion, gravity release, jitter, and focal plane misalignment as summarized in Table 3. Errors were estimated using mechanical, thermal, optical simulations, and technology development tests and meet STAR-X requirements.

Table 3. The XTA PSF error budget demonstrates the STAR-X design meets the 5 arcsec requirement based on analysis and test.

\begin{tabular}{|l|c|c|}
\cline { 2 - 3 } \multicolumn{1}{c|}{} & \multicolumn{2}{c|}{ Error (arcsec HPD) } \\
\cline { 2 - 3 } \multicolumn{1}{c|}{} & Budget & CBE \\
\hline Pointing Control Stability & $0.45(1.003 \sigma)$ & $0.21(0.533 \sigma)$ \\
\hline Focal Plane Misalignment & 1.54 & 0.81 \\
\hline Mirror Assembly Thermal Distortion & 0.75 & 0.33 \\
\hline Mirror Assembly Gravity Release & 0.50 & 0.20 \\
\hline Meta-shell Alignment and Bonding & 1.12 & 0.42 \\
\hline Mirror Alignment and Bonding & 2.24 & 1.68 \\
\hline Mirror Segment Fabrication & 2.55 & 2.06 \\
\hline Optical Design & 3.00 & 3.00 \\
\hline Total (RSS) & $\mathbf{5 . 0}$ & $\mathbf{4 . 1}$ \\
\hline
\end{tabular}

\section{CONCLUSIONS}

The STAR-X science goals are to 1) discover what powers the most violent explosions in the Universe 2) understand how black holes grow across cosmic time and mass scale and 3) measure how structure formation heats the majority of baryons in the Universe. This breakthrough science is enabled by the wide field of view, large effective area, and excellent uniform PSF of the XTA instrument. The only new technology in the XTA is the X-ray optics, which are based on polished single crystal silicon mirror segments aligned and bonded into meta-shell sub-assemblies. The X-ray optics technology is being matured by the Next Generation X-Ray Optics (NGXO) group at NASA GSFC with funding from the NASA ROSES Strategic Astrophysics Technology (SAT) program. The CCD detector system is based on heritage technology from ASCA, Chandra, Suzaku, and Pan-STARRS. The structural and thermal designs, contamination control approach, and error budget have all been developed to a level commensurate with the pre-Phase A mission study. 


\section{REFERENCES}

[1] Zhang, W. W., et al., "The survey and time-domain astrophysical research explorer (STAR-X)," Proc. SPIE 10397-25, (2017).

[2] Zhang, W. W., et al., "Silicon mirror segments, meta-shells, and mirror assemblies for x-ray astronomy," Proc. SPIE 10399-27, (2017).

[3] Saha, T. T., et al., “Optical design of the STAR-X telescope,” Proc. SPIE 10399-17, (2017).

[4] Riveros, R. E., et al., "Progress on the fabrication of lightweight single-crystal silicon x-ray mirrors," Proc. SPIE 1039928, (2017).

[5] Olds, C. R., Reese, R. P., "Composite structures for the advanced x-ray astrophysics facility (AXAF) telescope," Proc. SPIE 3356, (1998).

[6] O’Dell, S., et al., "Modeling contamination migration on the Chandra X-ray Observatory II," Proc. SPIE, 8859, (2013).

[7] Glenn, P., “Centroid detector assembly for the AXAF-I alignment test system,” Proc. SPIE, 2515, (1995). 